\title{
Use of LEDs for tuning of spectra of indoor luminaires
}

\begin{abstract}
Not so far ago the two most important characteristics of a light source to be used in indoor lighting were lighting efficiency and colour rendering index. First characteristic allow us to make lighting installation also energy efficient and second one assures that the lighting will be well accepted by the users or at least that the premises will look natural. Although both depend on light source spectra, it was very difficult to adapt spectra of the classical light sources to the needs of users. These changes with the introduction of power LEDs to the interior lighting. Today with use of white and monochromatic LEDs we can tailor spectra of luminaires to our needs. This approach can be used not only to obtain better energy efficiency and colour rendering index but also to adapt the spectra to the "non-visual needs" of users. The paper will show some approaches for the selection of the appropriate LEDs for tuning of spectra and the obtained results with different number of used LEDs in luminaire.
\end{abstract}

Keywords: indoor luminaires, light emitting diodes - LED, spectrum tuning.

\section{Introduction}

In last years we are facing huge developments of lighting technology in every aspects of our life. These developments are probably most noticeable in the field of light sources where light emitting diodes or LEDs managed to conquer the market in less than a decade. Compared with former light sources like incandescent lamps of fluorescent tubes, LEDs have their own unsurpassed characteristics like low energy use, high intensity, long life span and different spectra. Those are the reasons why LEDs caused a small revolution in lighting and photometry technology. Not just high power white LEDs but also high power monochromatic LEDs. They can be found today in many different devices like traffic signals, medical equipment, automotive lighting, decorative lighting and also in indoor lighting. As they are already available with many different peak wavelengths they can also be used in light sources with special spectrum of light. One possibility is a source with fixed spectrum for special purposes like white light sources with very high correlated colour temperature (CCT) e.g. used for treatment of seasonal affective disorder (SAD). The other possibilities include light sources with tunable spectrum.

\section{Light spectrum for indoor lighting}

For a long time spectrum of the light didn't play any important role in indoor lighting. In the time when only incandescent lamps were used, the spectrum was more or less the same with some differences in colour temperature. With the introduction of fluorescent lamps to the indoor lighting the possibility to choose spectrum (at least to some extend) occured for the first time. Different spectra were (and still are) available but selection was not done on the spectrum but on the correlated colour temperature (CCT) and colour rendering index ( $\mathrm{CRI}$ or $\mathrm{R}_{\mathrm{a}}$ ). The reason for that was that due to limited number of different product on market, it was not possible to choose a spectrum of the lamp but only a lamp with combination of CCT and CRI.

In last years a lot of research was done on influence of light spectrum on the human body and behaviour. Beside other results, it was found out that biologically speaking, warm white light (light with low CCT or light with expressed red part of the spectrum) has a relaxing effects on human organism whereas cold white light (light with high CCT or light with expressed blue part of the spectrum) boosts activity [1].

The research results about influence of light spectra on human body leaded to so called biologically active lighting or dynamic lighting. To be able to implement this kind of lighting it is necessary to use luminaires with tunable or at least changeable spectrum. With the fluorescent technology this is not so easy to achieve it as we need different sorts of lamps in the same luminaire. But with the LEDs such luminaires are much easy to realize. With help of different narrow band (monochromatic) and wide band (white or phosphor coated) LEDs it is possible to synthesize practically any possible spectrum in visible as well as also (to some expend) IR and UV range. So to get a luminaire with a tunable spectrum, different LEDs with different spectra need to be combined and afterwards controlled in a right way.

\section{Selection of LEDs}

There is a huge number of LEDs available on market today. They have different properties like electrical power, forward current, peak wavelength, FWHM (full width at half maximum), optical power or radiant flux, luminous flux, viewing angle... which makes the selection process very difficult. To reduce the number of options one should first consider the needed properties of the end product:

- as the tunable LED luminaire will be used for indoor lighting and not e.g. in lighting booth, the LEDs should have high output luminous flux so only the power LEDs should be used.

- If the luminaire should reproduce many different spectra, large number of LEDs with rather small FWHM should be used. On the other hand, if only some spectra should be reproduced (e.g. warm and cold white light), the selection of LEDs with wide FWHM could reduce the needed number of different LEDs.

To get good spectrum reproduction with different target spectra, the peek wavelengths (PWL) of LEDs should be as evenly distributed along the spectral range as possible.

In most cases, the tunable LED luminaire (TLL), which will be used for different purposes in different indoor environments, should have most of mentioned properties: high output luminous flux and possibility to reproduce many different spectra with best possible spectrum reproduction. That means that monochromatic power LEDs with rather narrow FWHM and with peek wavelengths equally distributed along the spectral range (usually between 380 $\mathrm{nm}$ and $780 \mathrm{~nm}$ ) should be used together with white (wide band) or phosphor coated LEDs.

If instead of more general LED luminaire, capable of reproducing large number of different spectra, the luminaire with just one spectrum or with small number of different output spectra should be build, the selection process of LEDs is different. In this case smaller number of LEDs can be used and selection can be made with optimization of sum of LEDs spectra as described in following section. 


\section{Spectrum optimization}

Under "spectrum optimization" one usually understands the adjustment of the output spectrum of tunable LED luminaire to closely match the target spectrum. On the other hand it could also mean the selection of needed LEDs to obtain the target spectrum of LED luminaire. In both cases the same procedures can be used.

First the target spectrum needs to be defined. This can be spectrum of one of CIE standard illuminants (e.g. A2856 or D65 standard illuminant spectrum) or any other arbitrary spectrum. It is practically impossible to fit the output spectrum of TLL completely to the target spectrum so the optimization criteria or goodness of fit need to be defined. In most cases the sum of squared differences between output spectrum of TLL and target spectrum is used as defined with equation 1.

$$
F=\sum_{l=380}^{780}\left(S_{T L L l}-S_{T \text { arg } e t l}\right)^{2}
$$

where: $S_{\text {Target I }}$ - value of target spectrum at wavelength I, $S_{T L L I}$ - value of TLL output spectrum at wavelength I

If one takes into consideration that the output spectrum is combination of spectra of used LEDs, the equation 1 can also be written as:

$$
F=\sum_{l=380}^{780}\left(\sum_{i=1}^{N}\left(a_{i} \cdot S_{L E D i, l}\right)-S_{T \arg e t l}\right)^{2}
$$

where: $S_{\text {Target I }}$ - value of target spectrum at wavelength I, $S_{L E D i, I}$ - value of spectrum of i-th LED at wavelength I,

$a_{i} \quad-$ multiplication factor proportional to the current through i-th LED,

$N \quad$ - number of LEDs used in TLL

There are different possibilities to find the values of $a_{1}$ coefficients which would minimize the optimization criteria function defined by equation 2. In most cases authors use some kind of iterative procedure [2,3]. One possibility is that next set of coefficient is calculated from previous one with the help of partial derivatives of the criteria function like in equation 3.

$$
a_{i}^{(n+1)}=a_{i}^{n}-b \cdot \frac{\partial F}{\partial a_{i}}
$$

where $b>0$ is small enough so that $F(a(n+1))<F(a(n))$.

The procedure - calculation of new values for multiplication factors $a_{i}$ can be repeated until the difference between criterion functions $F(a(n+1))$ and $F(a(n))$ is smaller than set threshold.

Use of such iterative approach always raises the question of starting values for coefficients $a_{i}$. One can start with all $a_{i}=0$ but in this case the procedure may take rather long time to find satisfactory solution. Probably the fastest way is to calculate the initial values with help of Gauss least square method like in [3] or [4]:

$$
A^{0}=\left(\Lambda^{T} \cdot \Lambda\right)^{-1} \cdot \Lambda^{T} \cdot S_{T \arg e t}
$$

where: $A^{0}$ - vector of initial values of $a_{i}$ coefficients, $\Lambda$ matrix with the spectra of all used LEDs, STarget $_{\text {- target }}$ spectrum
The problem of this method is that some of the calculated initial values of $a_{i}$ coefficient might have negative values. As these values represent relative values of nominal current through the LEDs, the negative values are not permissible. One possible solution is that these negative values are replaced by zeros, but also some methods with better results (regarding initial values of coefficients) are possible like one in [4].

Based on our experiences, the best way to obtain the initial values of $a_{i}$ coefficient is to use the Gauss least square method first on the full set of LEDs. If some of the calculated coefficients are negative, one LED with negative coefficient is excluded from the set and the Gauss method is repeated with so reduced set of LEDs. Once none of calculated $a_{i}$ coefficients is negative, the procedure can be stopped and coefficients for excluded LEDs are set to zero.

This gives rather good set of starting values of $a_{i}$ coefficients to be used with iterative procedure described before. The calculated initial values would, with the use of proper calculation procedure, give already the best fit solution if LED spectra would be linearly dependant on the LED forward current. Unfortunately this is not the case. The spectrum of LED (peek value, luminous intensity or luminous flux) does not change linearly with the forward current through the LED as well as peak wavelength, what makes the problem even more complicated. That is why an iterative procedure should be used after the calculation of initial values of coefficients $a_{i}$ to obtain better output spectrum of the tunable LED luminaire.

\section{Experiences with tunable LED light source}

To test the behavior of the multi LED luminaire and to test the optimization procedure a tunable LED lighting source was made at the University of Ljubljana, Faculty of Electrical Engineering. To enable the measurement of the output spectrum and to test its properties, the tunable light source was not made in shape of luminaire but rather in shape of lighting booth.

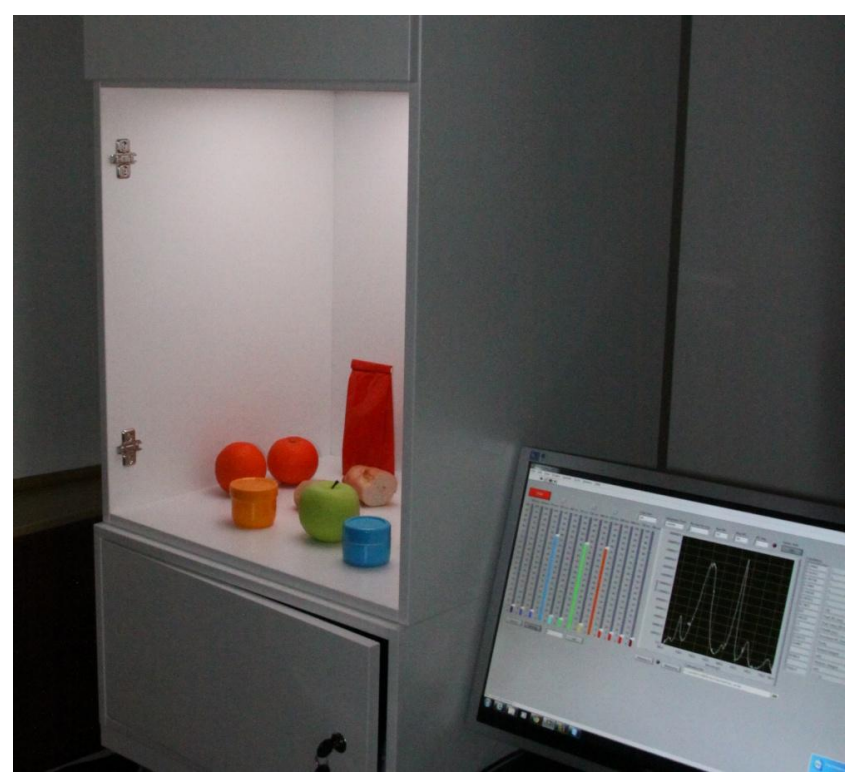

Fig.1. Test tunable LED light source at University of Ljubljana

Altogether 13 different narrow band power LEDs were used in the source. Standard LEDs mounted on a hexagonal aluminum package were placed in the upper part of the box, so that the light reflects from the ceiling. In the lower part of the box a mixed spectrum is reached with an almost uniform illuminance on the floor. The basic data of 
the LEDs is listed in the table 1 together with their commercial designations.

Table 1. Basic parameters of power LEDs used in a tunable LED light source where: Dsg - designation, If - nominal forward current, PWL - nominal peak wavelength, PWLm - measured PWL at nominal current

\begin{tabular}{|l|c|c|c|}
\hline Dsg & If $(m A)$ & PWL $(n m)$ & PWLm (nm) \\
\hline H2A1-H395 & 350 & 395 & 368 \\
\hline H2A1-H410 & 350 & 410 & 388 \\
\hline H2A1-H435 & 350 & 435 & 404 \\
\hline H2A1-H470 & 350 & 470 & 423 \\
\hline H2A1-H490 & 350 & 490 & 431 \\
\hline H2A1-H515 & 350 & 515 & 455 \\
\hline H2A3-H530 & 700 & 530 & 466 \\
\hline LXHL-ML1D-590 & 350 & 590 & 491 \\
\hline H2A3-H625 & 700 & 625 & 498 \\
\hline H2A1-H650 & 350 & 650 & 514 \\
\hline H2A1-H680 & 350 & 680 & 531 \\
\hline H2A1-H720 & 350 & 720 & 520 \\
\hline H2A1-H760 & 350 & 760 & 590 \\
\hline
\end{tabular}

The spectra of the used LEDs, measured with the nominal current are shown on figure 2 .

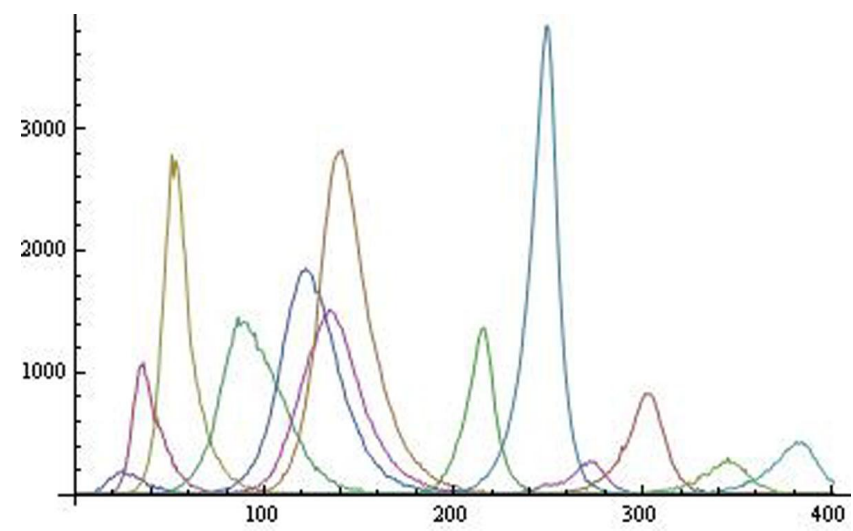

Fig.2. Measured spectra of the LEDs used in a tunable light source

A special control program, running on a LabView platform was developed to calculate optimization coefficient for different target spectra. Figure 3 shows the optimized solution for CIE A2856 spectrum and figure 4 shows the solution for CIE D65 spectrum. From both pictures it can be seen that LED spectra do not overlap enough to reproduce the target spectra very accurately.

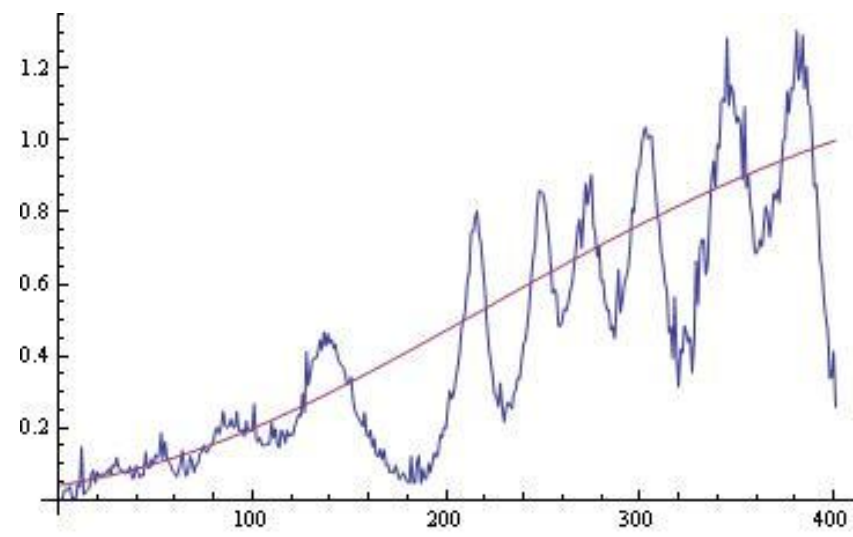

Fig.3. Optimized reproduction of CIE A2856 spectrum with 13 LEDs in tunable LED light source

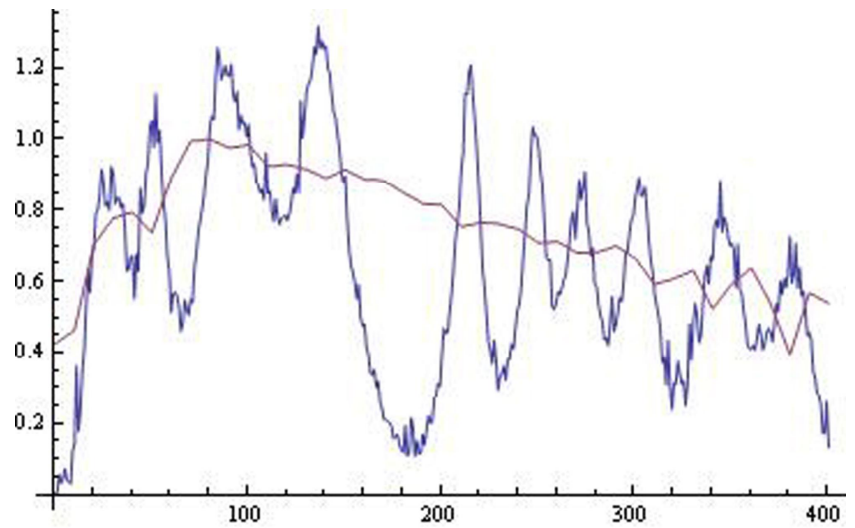

Fig.4. Reproduction of CIE D65 spectrum with LED light source

On the other hand, the spectrum is much closer to the target spectrum as spectrum of the fluorescent lamp with the same CCT and CRI.

Although the optimized spectrum may not always have the same CCT (and CRI) as the target spectrum, both parameters can be changed with some rather small changes of forward current of individual LEDs.

\section{Conclusions}

As already confirmed by some commercial products, tunable spectrum luminaires can be realized with rather small number of different LEDs and suitable control module. With proper optimization process the output spectrum of such luminaire can be very close to the target one and have the same CCT and very good CRI. It is also very easy to change the output spectrum and so also CCT and/or CRI. But there are still some practical problems which need to be overcome. One is shift in spectrum of LEDs at different forward currents or temperatures of $p-n$ junction which can cause unwanted change in output spectrum. With laboratory tunable LED sources this can be overcome with measurement of output spectrum and closed loop operation of the LED control. But due to the high price of such solution, for the tunable LED luminaires some other approach needs to be found.

\section{REFERENCES}

[1] licht.de, Good Lighting for a Better Learning Environment, Fördergemeinschaft Gutes Licht, Frankfurt am Main, ISBN-Nr. 978-3-926193-70-4

[2] FRYC I., BROWN S.W., EPPELDAUER G.P., OHNO Y. 2005. LED-based spectrally tunable source for radiometric, photometric, and colorimetric applications, Optical Engineering, 44(11), 111309, November 2005

[3] BIZJAK G., LINDEMANN M., SPERLING A., SAUTER G. 2010. Tunable LED colour source. Proceedings of the CIE Expert Symposium on Spectral and Imaging Methods for Photometry and Radiometry. Vienna. CIE, 2010, 99-102

[4] C-C WU, N-C HU, Y-C FONG, H-C HSIAO AND S-L HSIAO. 2012. Optimal pruning for selecting LEDs to synthesize tunable illumination spectra. Lighting Research and Technology 2012 44: 484

Authors: prof. dr. Grega Bizjak, Laboratory of Lighting and Photometry, Faculty of Electrical Engineering, University of Ljubljana, Trzaska cesta 25, SI-1000 Ljubljana, Slovenia grega.bizjak@fe.uni-lj.si

dr. Kobav Matej Bernard, Laboratory of Lighting and Photometry, Faculty of Electrical Engineering, University of Ljubljana, Trzaska cesta 25, SI-1000 Ljubljana, Slovenia

matej.kobav@fe.uni-lj.si 